\title{
Laparoscopic surgery: an effective and safe surgical method of pediatric inguinal hernia repair
}

\author{
Eun Jung Kim, Chaeyoun Oh, Jun Won Um \\ Department of Surgery, Korea University Ansan Hospital, Ansan, Korea
}

Purpose: Inguinal hernia (IH) repair is very commonly performed in children. While open repair (OR) is the standard approach, laparoscopic repair is increasingly used. This study was aimed to investigate safety and feasibility of laparoscopic repair of pediatric IH compared to OR.

Methods: We retrospectively enrolled 105 pediatric patients with IH repair between January 2011 and October 2019. The laparoscopic procedures performed were laparoscopic percutaneous extraperitoneal closure (LPEC), and three-port mini-laparoscopic repair (TLR). The OR was performed as per usual technique.

Results: Thirty-nine patients underwent OR, 16 LPEC, and 50 TLR. The preoperative laterality of IH was 45 patients (42.9\%) on the right side, $50(47.6 \%)$ on the left side, and $10(9.5 \%)$ on both sides. It was, however, diagnosed postoperatively in 27 patients (25.7\%) on the right side, 38 (36.2\%) on the left side, and 40 (38.1\%) on both sides. Of the 63 patients who presented with unilateral IH in the laparoscopic groups, 32 (50.8\%) had synchronous contralateral patent process vaginalis (PPV) which were simultaneously repaired. This was significantly more common in children under 3 years of age. Operative time in unilateral or bilateral repair was significantly shorter in the laparoscopic repair groups $(p<0.001)$. Ipsilateral recurrence was not observed in any group. Metachronous contralateral $\mathrm{IH}$ occurrence was not significantly different between groups.

Conclusion: Laparoscopic IH repair may have benefit in terms of shorter operation time and diagnosis of unpredicted contralateral PPV compared to OR.

Keywords: Inguinal hernia, Pediatrics, Herniorrhaphy, Laparoscopy

This is an Open Access article distributed under the terms of the Creative Commons Attribution Non-Commercial License (http:// creativecommons.org/licenses/by-nc/4.0/) which permits unrestricted non-commercial use, distribution, and reproduction in any medium, provided the original work is properly cited.
Received August 11, 2021

Revised 1st October 5,2021 2nd October 22, 2021

Accepted October 31, 2021

Corresponding author Jun Won Um

Department of Surgery, Korea University Ansan Hospital, 123 Jeokgeum-ro, Danwon-gu, Ansan 15355 , Korea

Tel: +82-31-412-4830

Fax: +82-31-412-4807

E-mail: junwonum@korea.ac.kr ORCID:

https://orcid.org/0000-0002-0506-0235
Copyright (C) The Korean Society of Endoscopic and Laparoscopic Surgeons.

\section{INTRODUCTION}

Inguinal hernia $(\mathrm{IH})$ repair is the most common surgery performed in children. In children under the age of 18 , the incidence of $\mathrm{IH}$ is $0.8 \%$ to $5 \%$ at full term and up to $30 \%$ with low birth weight and premature births [1]. Pediatric IH is caused by persistent patency of the vaginal process, unlike $\mathrm{IH}$ in adults, which is due to weakness of the abdominal musculature. Therefore, prosthetic mesh reinforcement is necessary in adults, whereas high ligation is sufficient in children [2].
High ligation using open repair (OR) was described over 100 years ago, and the recurrence rate in recently published reports is very low, ranging from $0 \%$ to $6 \%[3,4]$. However, complications such as spermatic cord injury, vas deference injury, hematoma, surgical site infection, iatrogenic cryptorchidism, and testicular atrophy can be seen [5]. The laparoscopic approach was first reported for pediatric IH repair in 1992 [6]. Although OR is still widely performed, laparoscopic IH repair is being increasingly used in many institutions, even in neonates, where minimally invasive surgery is mainstream. It has been recently reported 
that, compared to OR, laparoscopic bilateral $\mathrm{IH}$ repair is associated with shorter operation time and fewer wound infections in infants [7]. This study was aimed to investigate safety and feasibility of laparoscopic repair of pediatric IH compared to OR in a single center.

\section{MATERIALS AND METHODS}

This study was conducted on 105 children and adolescents aged $<19$ years who underwent IH surgery at Korea University Ansan Hospital from January 2011 to October 2019. We compared OR and laparoscopic repair of pediatric $\mathrm{IH}$ performed in a single center and further analyzed the results of two laparoscopic techniques, laparoscopic percutaneous extraperitoneal closure (LPEC) and three-port mini-laparoscopic repair (TLR). It is our policy to conduct only one outpatient follow-up visit approximately one week after the operation, with no further follow-up unless indicated. Therefore, all subjects were contacted via telephone interviews to determine their recent status. Telephone interviews were attempted in October 2019 with 105 patients, and 29 out of 39 patients $(74.4 \%)$ in the OR group responded. All 16 patients in the LPEC group responded (100\%), and 46 out of 50 patients (92.0\%) in the TLR group responded. The data were collected retrospectively through medical charts, such as surgical records and anesthesia records.

OR was performed by two surgeons. LPEC has been performed since 2015, and TLR has been performed since 2018. In OR, high ligation was performed only on the symptomatic side, whereas in laparoscopic repair, bilateral repairs were performed when contralateral patent processus vaginalis (PPV) was confirmed. General anesthesia was used for all operations. For the OR, a 1 to 2 $\mathrm{cm}$ incision was made in the symptomatic inguinal area, the her- nia sac was retrieved, the cord structure was detached, and high ligation was performed. LPEC was performed as previously reported [8]. A 5-mm camera was inserted through the umbilicus, and one 3-mm port was placed in the suprapubic area, through which one assisted laparoscopic instrument was inserted. After checking the patency of the processus vaginalis, an incision of approximately 1 to $2 \mathrm{~mm}$ was performed above it. Subsequently, preperitoneal sutures were placed first on the medial side, from 12 o'clock to 6 o'clock of the internal inguinal ring, using a 21gage spinal needle. At that time, an assisted instrument was used to direct the vas deference and gonadal vessels down the suture line. The needle was removed after coming out in the direction of 6 o'clock, and the 4-0 prolene was pushed down to form the loop. The same process then started in the opposite direction, from 12 o'clock to 6 o'clock, with the needle coming out at 6 o'clock. The sufficiently delivered prolene was caught with a loop and came out, and a tie was carefully performed at the incision site. At that time, the operation was concluded after checking whether the internal inguinal ring was sufficiently closed through the camera (Fig. 1). TLR was performed using the laparoscopic sac transection and peritoneal closure method [9]. We performed the procedure using a 3-mm camera and two 3-mm instruments. After complete transection of the hernia sac at the level of the internal inguinal ring, the peritoneum around the ring was sutured with a 4-0 prolene suture to close it (Fig. 2).

The nonparametric variance analysis was performed using the Mann-Whitney $U$ test. Comparisons between variables were evaluated using the chi-square test. Statistical significance was set at $p<0.05$. Statistical analysis was performed using IBM SPSS version 20 (IBM Corp., Armonk, NY, USA).
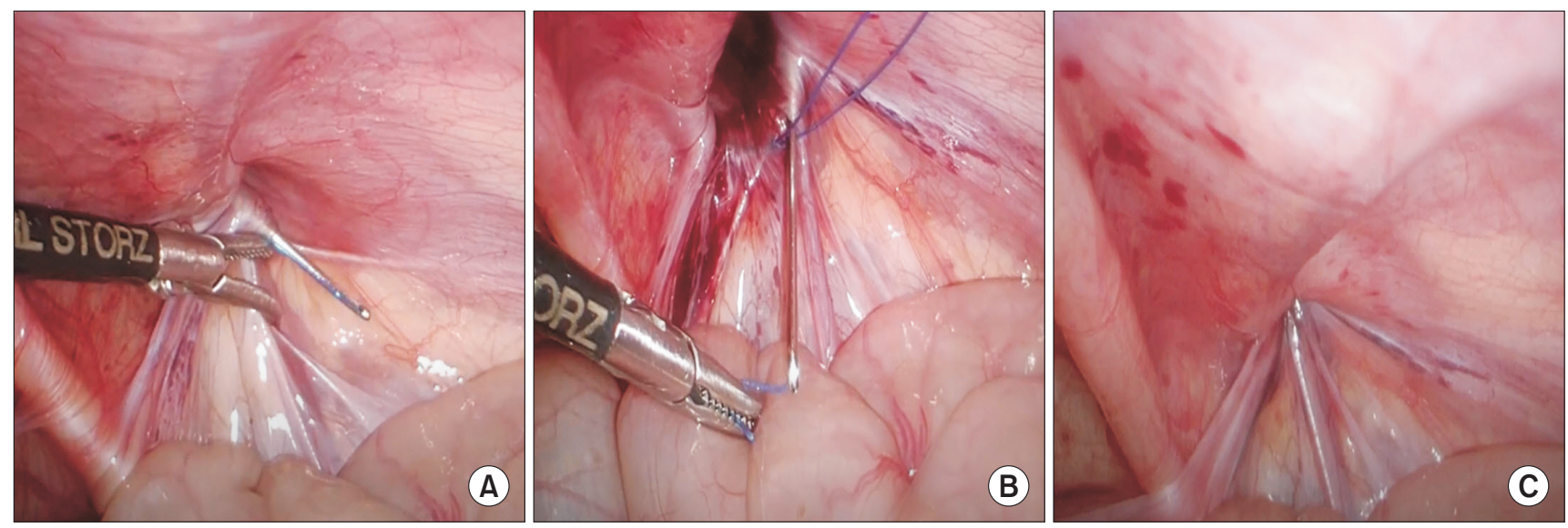

Fig. 1. Laparoscopic percutaneous extraperitoneal closure. (A) Preperitoneal suturing performed first on the medial side from 12 o'clock to 6 o'clock of the internal inguinal ring using a 21-gage spinal needle. (B) Suturing in the opposite direction from 12 o'clock to 6 o'clock, with the needle coming out into the loop at 6 o'clock. (C) Closure of the internal inguinal ring. 

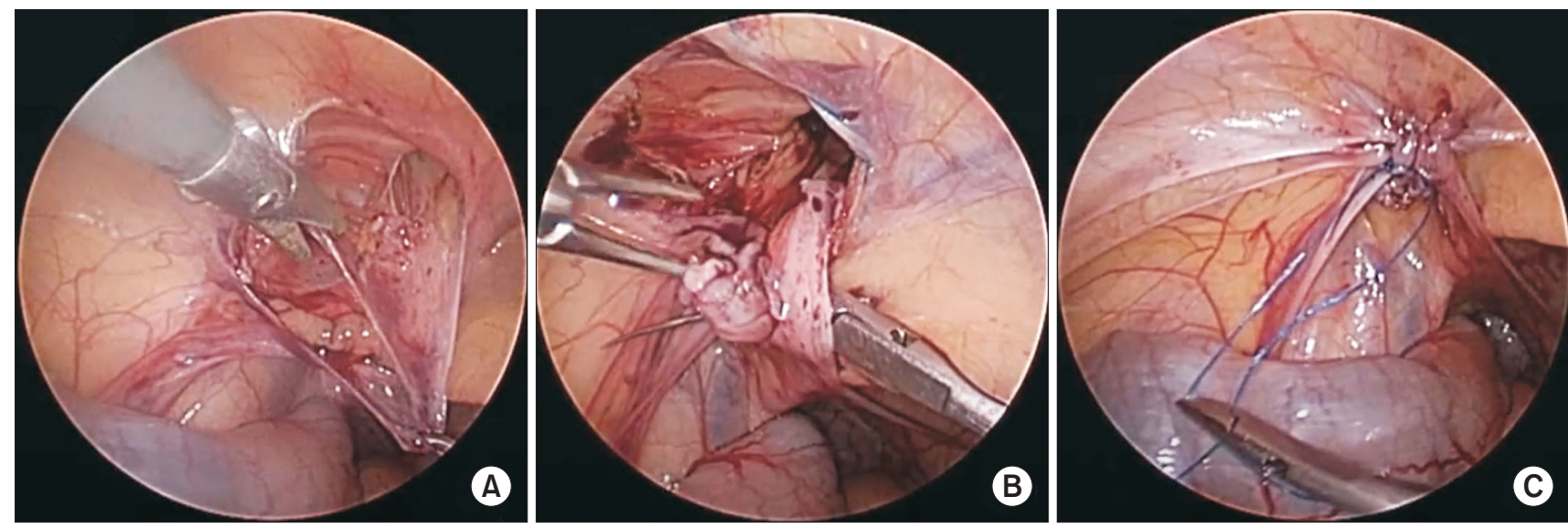

Fig. 2. Three-port mini-laparoscopic repair. (A) The hernia sac is transected at the level of the internal inguinal ring. (B) Suturing the peritoneum around the internal inguinal ring with a purse-string suture. (C) Closure of the internal inguinal ring.

Table 1. Patient characteristics

\begin{tabular}{|ccccc|}
\hline \multicolumn{1}{c}{ Characteristic } & Total & Open & Laparoscopy & $p$ value \\
\hline No. of patients & 105 & 39 & 66 & $>0.999$ \\
\hline Male sex & $77(73.3)$ & $29(74.4)$ & $48(72.7)$ & $>0.999$ \\
\hline Age at operation (mo) & $17.2(0.8-183.5)$ & $17.1(0.8-136.7)$ & $20.2(0.8-183.5)$ & 0.686 \\
$<3$ mo & $30(28.6)$ & $12(30.8)$ & $18(27.3)$ & 0.824 \\
$<1$ yr & $45(42.8)$ & $18(46.2)$ & $27(40.9)$ & 0.684 \\
$<3$ yr & $65(61.9)$ & $24(61.5)$ & $41(62.1)$ & $>0.999$ \\
$\geq 3$ yr & $40(38.1)$ & $15(38.5)$ & $25(37.9)$ & $>0.999$ \\
Weight at operation (kg) & $10.5(3.08-55)$ & $10.2(3.08-42)$ & $11(3.2-55)$ & 0.907 \\
$<5$ & $21(20.0)$ & $8(20.5)$ & $13(19.7)$ & $>0.999$ \\
$<10$ & $47(44.8)$ & $19(48.7)$ & $28(42.4)$ & 0.549 \\
$\geq 10$ & $58(55.2)$ & $20(51.3)$ & $38(57.6)$ & 0.549 \\
Initial symptom & & & & \\
Inguinal swelling & $104(99)$ & $39(100)$ & $65(98.5)$ & $>0.999$ \\
Vomiting & $2(1.9)$ & $1(2.6)$ & $1(1.5)$ & $>0.999$ \\
Fever & $3(2.9)$ & $0(0)$ & $3(4.5)$ & 0.293 \\
Hydrocele & $21(20.0)$ & $11(28.2)$ & $10(15.2)$ & 0.132 \\
Major comorbidities) & $5(4.8)$ & $1(2.6)$ & $4(6.1)$ & 0.649 \\
Follow-up (mo) & $23.6(0.5-106.7)$ & $83.8(11.0-106.7)$ & $8.7(0.5-58.4)$ & $<0.001$ \\
\hline
\end{tabular}

Values are presented as number only, number (\%), or median (range).

${ }^{a}$ Atrial septal defect, 1; ventricular septal defect, 1; patent ductus arteriosus, 1; coarctation of aorta, 1; tetralogy of Fallot, 1.

\section{RESULTS}

Of the 105 patients, $73.3 \%$ were male. The median age and median weight at the time of surgery were similar in the OR and laparoscopy groups. Most of the patients presented with inguinal swelling, and there was no significant difference between the two groups in the number of patients with major comorbidities. The median duration of follow-up after surgery was 23.6 months, and the recently performed laparoscopy group was significantly shorter than the OR group (Table 1). In subanalysis within the laparoscopic group, there were 16 patients in the LPEC group and 50 patients in the TLR group. There were 16 patients in the LPEC 
Table 2. Operation findings

\begin{tabular}{|c|c|c|c|c|}
\hline Variable & Total & Open & Laparoscopy & $p$ value \\
\hline No. of patients & 105 & 39 & 66 & \\
\hline \multicolumn{5}{|l|}{ Side of initial presentation } \\
\hline Right & $45(42.9)$ & $15(38.5)$ & $30(45.5)$ & 0.544 \\
\hline Left & $50(47.6)$ & $17(43.6)$ & $33(50.0)$ & 0.551 \\
\hline Bilateral & $10(9.5)$ & $7(17.9)$ & $3(4.5)$ & 0.037 \\
\hline \multicolumn{5}{|l|}{ Side at operation } \\
\hline Right & $27(25.7)$ & $15(38.5)$ & $12(18.2)$ & 0.036 \\
\hline Left & 38 (36.2) & $17(43.6)$ & $21(31.8)$ & 0.294 \\
\hline Bilateral & $40(38.1)$ & $7(17.9)$ & $33(50.0)$ & 0.002 \\
\hline Right to bilateral & $20(19.0)$ & None & $20(30.3)$ & \\
\hline Left to bilateral & $12(11.4)$ & None & $12(18.2)$ & \\
\hline \multicolumn{5}{|l|}{ Operation time (min) } \\
\hline Unilateral & $43.5 \pm 14.7$ & $49.7 \pm 15.4$ & $37.5 \pm 11.2$ & $<0.001$ \\
\hline Bilateral & $50.1 \pm 22.1$ & $86.3 \pm 22.2$ & $42.4 \pm 12.6$ & $<0.001$ \\
\hline Postoperative stay (day) & $0.73 \pm 0.7$ & $0.9 \pm 0.55$ & $0.64 \pm 0.71$ & 0.018 \\
\hline
\end{tabular}

Values are presented as number only, number $(\%)$, or mean \pm standard deviation.

group and 50 patients in the TLR group. The median age and median weight at the time of surgery were significantly lower in the LPEC group (age, 8.5 months [range, 1.3-54.4 months]; weight, $7.6 \mathrm{~kg}$ [range, $3.2-17.6 \mathrm{~kg}$ ]) compared to the TLR group (age, 30.5 months [range, $0.8-183.5$ months]; weight, $13.1 \mathrm{~kg}$ [range, $3.3-55.0 \mathrm{~kg}] ; p=0.019$ and $p=0.048)$.

The initial presentation was on the right side in $42.9 \%$ of cases, on the left side in $47.6 \%$, and on both sides in $9.5 \%$. Among the patients who underwent laparoscopic surgery, contralateral PPV was found on the left side in 20 of 30 patients (66.7\%) whose initial presentation was on the right, and on the right side in 12 of 33 patients (36.4\%) whose initial presentation was on the left. That is, out of 63 patients who initially presented for unilateral laparoscopic repair, 32 patients (50.8\%) were found to have synchronous contralateral PPV which was simultaneously repaired. Among the patients in the group who underwent laparoscopic surgery, three patients had a history of bilateral inguinal swelling, but two patients underwent unilateral repair because PPV was confirmed only on the right side. Operation time was significantly shorter in the laparoscopy group for both unilateral and bilateral repairs, and the postoperative hospital stay was also significantly shorter in the laparoscopy group than OR group (Table 2). In subanalysis within the laparoscopic group, operation time was significantly shorter in the TLR group for both unilateral and bilateral repairs than LPEC group (unilateral, $33.7 \pm 8.4$ minutes vs. $47.7 \pm 11.8$ minutes; bilateral, $37.0 \pm 6.0$ minutes vs. $62.9 \pm 8.9$ minutes; $p<$ 0.001 and $p<0.001$.
Table 3. Contralateral patent processus vaginalis in laparoscopic repair group

\begin{tabular}{|crc|}
\hline \multicolumn{1}{|c}{ Variable } & No. (\%) & $p$ value \\
\hline Sex & & 0.272 \\
Male & $21 / 48(43.8)$ & \\
Female & $11 / 18(61.1)$ & \\
Age & & 0.010 \\
$<3 \mathrm{mo}$ & $11 / 18(61.1)$ & \\
$3 \mathrm{mo}-6 \mathrm{mo}$ & $4 / 5(80.0)$ & \\
$6 \mathrm{mo}-1 \mathrm{yr}$ & $2 / 4(50.0)$ & \\
$1 \mathrm{yr}-3 \mathrm{yr}$ & $10 / 14(71.4)$ & \\
$3 \mathrm{yr}-5 \mathrm{yr}$ & $2 / 12(16.7)$ & \\
$\geq 5 \mathrm{yr}$ & $3 / 13(23.1)$ & \\
\hline
\end{tabular}

In the laparoscopic repair groups, $43.8 \%$ of males and $61.1 \%$ of females had a contralateral PPV. This finding appeared to be more frequent in females, but the difference was not significant. By age, contralateral PPV was found in $80 \%$ of patients aged from 3 to 6 months, and in 71.4\% of patients aged from 1 to 3 years. These results suggest that contralateral PPV tends to decrease significantly after 3 years of age (Table 3 ).

No ipsilateral recurrence occurred during the postoperative follow-up period. Surgical site infection was observed in one patient (1.5\%) after laparoscopic surgery. Ten patients $(9.5 \%)$ were 
Table 4. Postoperative complications and occurrence of metachronous contralateral inguinal hernia

\begin{tabular}{|c|c|c|c|c|}
\hline Variable & Total & Open & Laparoscopy & $p$ value \\
\hline No. of patients & 105 & 39 & 66 & - \\
\hline Recurrence & $0(0)$ & $0(0)$ & $0(0)$ & - \\
\hline Surgical site infection & $1(1.0)$ & $0(0)$ & $1(1.5)$ & $>0.999$ \\
\hline Hydrocele & $10(9.5)$ & $3(7.7)$ & $7(10.6)$ & 0.741 \\
\hline Hematoma & $2(1.9)$ & $2(5.1)$ & $0(0)$ & 0.136 \\
\hline Seroma & $2(1.9)$ & $0(0)$ & $2(3.0)$ & 0.529 \\
\hline Total $\left.\right|^{a)}$ & $14(13.3)$ & $5(12.8)$ & $9(13.6)$ & $>0.999$ \\
\hline Occurrence of metachronous contralateral inguinal hernia & $3(2.9)$ & $2(5.1)$ & $1(1.5)$ & 0.554 \\
\hline
\end{tabular}

Values are presented as number only or number (\%).

${ }^{a}$ Multiple complications could occur in the same patients.

found to have hydrocele after surgery, but there was no significant difference between groups. Of the 105 patients, three (2.9\%) experienced metachronous contralateral IH occurrence after surgery. It occurred in two of the OR group (5.1\%) and one of the laparoscopic repair groups (1.5\%), but the difference was not significant $(p=0.554)$. It occurred at 24.03 months, and 21.7 months after surgery in the two OR group patients, and at 2 months after surgery in the one LPEC patient (Table 4).

\section{DISCUSSION}

The operative time was shorter in the laparoscopic repair groups. Contralateral PPV was confirmed in $50.8 \%$ of patients who underwent laparoscopic repair for unilateral inguinal swelling, and the probability of such was higher in patients under 3 years of age. Laparoscopic IH repair may have benefit in terms of shorter operation time and diagnosis of unpredicted contralateral PPV compared to OR especially in younger patients.

Almost all IHs in children are congenital indirect hernias that occur through the processus vaginalis, the anatomical path created before birth, through which the testicles descend from the abdomen to the scrotum. The exact timing of the processus vaginalis closure is not clear. Some studies report that $80 \%$ to $100 \%$ of infants have a PPV at birth, with most of them closing within 6 months of age [10]. It is not well known why the processus vaginalis is not obliterated. Failure of the surrounding smooth muscle cells to undergo apoptosis when the testicles descend has been reported as a cause [11]. It has also been suggested that a decrease in the calcitonin gene-related protein release from the genitofemoral nerve can lead to hernia and scrotal edema after birth [12]. In females, it is the round ligament supporting the uterus that descends through the processus vaginalis, which, if persistently patent, is called the canal of Nuck, and can lead to hernia development [13].
$\mathrm{OR}$ is the traditional surgical method of hernia repair in children, and it is still widely practiced [14]. It is fast, safe, and can be done as outpatient. It has, however, a recurrence rate of about $1 \%$ to $5 \%$ depending on the skill level of the surgeon, the age of the patient, and the accompanying disease [15]. The introduction of laparoscopic repair in the 1990s was a milestone in surgical repair of pediatric hernias. In 1995, Takahara et al. [8] reported on a surgical method called LPEC, and since then, there have been several reports on its safety and efficacy $[14,16]$. In one systematic review comparing open and laparoscopic repair in children, the operation time was 30.1 minutes for OR and 23.7 minutes for laparoscopic repair in unilateral $\mathrm{IH}$, with no statistical difference between the two $(p=0.33)$. However, in the case of bilateral hernias, the laparoscopic operation time was significantly shorter than the OR time (46.1 minutes vs. 30.9 minutes, $p=0.01$ ) [7]. These findings have been reproduced in several other studies [17] and suggest that laparoscopic surgery is more efficient in terms of operation time than OR in pediatric IH repair.

In the same systematic review, the recurrence rate was $1.6 \%$ for OR and 1.4\% for laparoscopic repair with no statistical difference between the two techniques $(p=0.66)[7]$. However, the rate of complications such as wound infection, hydrocele, iatrogenic cryptorchidism, and testicular atrophy was higher in OR than in laparoscopic repair $(2.7 \%$ vs. $0.9 \%, p=0.001)$. In a study conducted by Ho et al. [17], there was no statistical difference between $\mathrm{OR}$ and laparoscopic repair in terms of postoperative recurrence rate and wound infection. However, postoperative scrotal swelling occurred more frequently in the OR group $(p=0.006)$. In other studies, metachronous contralateral hernia, surgical site infection, and scrotal swelling occurred significantly less with laparoscopic repair [18]. These results suggest that laparoscopic repair may be preferable, compared to OR, because of few complications such as cryptorchidism and testicular atrophy. This is probably because the visualization of cord structures is good, and 
dissection can be made safer. In addition, the laparoscopic dissection field is limited to the peritoneal layer, so the vas deferens is less likely to be injured [7].

In addition to a shorter operation time and fewer surgical complications, laparoscopic repair has many other advantages. Laparoscopic repair is a minimally invasive surgery associated with less pain, quick return to daily life, and excellent cosmetic outcomes [16]. In one study, hospital stay and recovery time were overall similar for OR and laparoscopic repair, but patients who underwent LPEC had fewer complications and shorter unilateral surgery time, whereas patients who received TLR had shorter hospital stays [19].

In a study by Korkmaz and Güvenç [16], which compared three-port conventional laparoscopic repair and LPEC, the operation time was seemed shorter in the LPEC group in unilateral hernia repair and was approximately the same in the two groups in bilateral repair. There was no recurrence in the LPEC group and two recurrences (4.3\%) in the three-port conventional laparoscopic repair group, which happened during the learning curve period. In the LPEC group, there were cases of inferior epigastric vessel injury resulting in OR and hydrocele formation after surgery. However, no complications occurred in the threeport conventional laparoscopic repair group. In our study, the operative time was shortest in the TLR group for both unilateral and bilateral repairs $(p<0.001)$. Metachronous contralateral $\mathrm{IH}$ occurred in one case $(6.2 \%)$ in the LPEC group, and none in the TLR group.

In this study, synchronous contralateral PPV was found in $50.8 \%$ of patients when laparoscopic repair was performed for unilateral inguinal swelling. In these cases, contralateral repair was performed simultaneously. There is controversy over contralateral groin exploration in infants and children with unilateral indirect IH [20]. The purpose of contralateral exploration is to prevent hernia from occurring later. Disadvantages of contralateral exploration include the possibility of damage to spermatic cord vessels, vas deferens, and testis, and an increase in operation time and anesthesia time [21]. Because contralateral PPV may not always progress to metachronous contralateral IH [22], its repair has been reported to be unnecessary [23]. However, contralateral exploration can avoid the risk of incarceration, additional surgical costs, additional anesthesia, and parental worries [24]. Because the opposite side can be explored without the risk of significant damage to the vas and vessels, pediatric surgeons are increasingly using laparoscopic exploration [25] to detect and repair contralateral PPV [26]. Rather than routinely performing contralateral exploration, several studies of IHs in children have recommended age-specific exploration [22,27]. According to Holcomb et al. [28], bilateral IHs occurred in $50 \%$ of children younger than 1 year, $45 \%$ of children younger than 2 years, and $37 \%$ of those younger than 5 years suggesting that the younger they were, the more common a bilateral IH was. After unilateral hernia correction, $5 \%-20 \%$ of patients had hernias developed on the contralateral side, and repair was needed. In our study, contralateral PPV in patients who underwent laparoscopic surgery for a unilateral indication was found in $80 \%$ of patients aged from 3 to 6 months and $71.4 \%$ of patients aged from 1 to 3 years, but in only $20 \%$ of those over 3 years old. Therefore, we believe that it is better to simultaneously repair contralateral PPV by a laparoscopic technique in patients under 3 years of age with unilateral symptoms.

The recurrence rate in pediatric hernia surgery is reported to be $0 \%$ to $6 \%$ in OR, and $0 \%$ to $15.5 \%$ in laparoscopic repair, and recurrence almost always occurs within 1 year after surgery [29]. In our study, there was no ipsilateral recurrence in the three groups during the median follow-up period of 23.6 months. In two patients who underwent $\mathrm{OR}$, metachronous contralateral IH occurred after 24.03 months and 21.7 months; whereas in one patient who underwent LPEC, metachronous contralateral IH occurred 2 months after surgery, and it was due to a small contralateral PPV which was missed. Sumida et al. [30] noted the importance of careful screening to reduce the occurrence of metachronous contralateral hernia in LPEC.

This study has some limitations. This institution was not a specialized pediatric surgical center until 2017. Therefore, the number of pediatric IH was not large. As a result, the study enrollment period became long, and after a pediatric surgeon joined in 2018, the number of pediatric laparoscopic surgery suddenly increased. This is a limitation of this study since the heterogeneity of the enrollees may have acted as a bias in the interpretation of the surgical outcome. In the laparoscopy group, the median duration of follow-up was short enough to be less than 1 year. Since such a short period is not sufficient to evaluate surgical complications, it is possible that the complications in the laparoscopy group were underestimated. In this study, postoperative hospital stay was shorter in the laparoscopic repair group than OR group, which may seem to suggest that laparoscopic repair allows faster return to daily life due to less postoperative pain and faster recovery than OR. However, this study had limitation in enrolling patients over 9 years. As medical care and technology advance during this period, the policy of surgeons and hospitals for 'hospital stay' changes. In this institution, both OR and laparoscopic surgery have recently been aimed at day surgery (shorter hospital stay). As mentioned earlier, LPEC has been performed in this institution since 2015, and TLR has been performed since 2018. Therefore, the postoperative hospital stay in this study would not have much meaning in the interpretation of the results due to the bias caused by the long study period.

In conclusion, the operative time of IH repair was shorter in the laparoscopic repair groups than in the OR group; metachronous contralateral IH occurred in $5.1 \%$ of the OR group and $1.5 \%$ of the laparoscopic repair group, but the difference was not sig- 
nificant. Contralateral PPV was confirmed in $50.8 \%$ of patients who underwent laparoscopic repair for unilateral inguinal swelling, and the probability of finding synchronous contralateral PPV was significant in patients under three years of age. Laparoscopic IH repair is safe and effective and can be used to detect synchronous contralateral PPV especially in younger patients, thus preventing metachronous contralateral $\mathrm{IH}$ occurrence.

\section{NOTES}

\section{Ethical statements}

This study was performed in accordance with the Declaration of Helsinki. This study was approved by the Institutional Review Board of Korea University Ansan Hospital (No. 2021AS0191), and informed consent was waived due to its retrospective nature.

\section{Authors' contributions}

Data curation, Formal analysis, Investigation, Visualization, Methodology, Project administration: EJK, CO

Writing-original draft: EJK, CO

Writing-review \& editing: CO, JWU

All authors read and approved the final manuscript.

\section{Conflict of interest}

All authors have no conflicts of interest to declare.

\section{Funding/support}

None.

\section{ORCID}

Eun Jung Kim, https://orcid.org/0000-0001-7521-0828

Chaeyoun Oh, https://orcid.org/0000-0001-9734-0869

Jun Won Um, https://orcid.org/0000-0002-0506-0235

\section{REFERENCES}

1. Burgmeier C, Dreyhaupt J, Schier F. Comparison of inguinal hernia and asymptomatic patent processus vaginalis in term and preterm infants. J Pediatr Surg 2014;49:1416-1418.

2. Mouravas V, Sfoungaris D. The etiology of indirect inguinal hernia in adults: congenital, acquired or both? Hernia 2015;19:1037-1038.

3. Esposito C, Giurin I, Alicchio F, et al. Unilateral inguinal hernia: laparoscopic or inguinal approach. Decision making strategy: a prospective study. Eur J Pediatr 2012;171:989-991.

4. Nah SA, Giacomello L, Eaton S, et al. Surgical repair of incarcerated inguinal hernia in children: laparoscopic or open? Eur J Pediatr Surg 2011;21:8-11

5. Esposito C, Montinaro L, Alicchio F, et al. Technical standardization of laparoscopic herniorraphy in pediatric patients. World J Surg 2009;33:1846-1850.

6. Lobe TE, Schropp KP. Inguinal hernias in pediatrics: initial experience with laparoscopic inguinal exploration of the asymptomatic contralateral side. J Laparoendosc Surg 1992;2:135-141.

7. Esposito C, St Peter SD, Escolino M, Juang D, Settimi A, Holcomb GW 3rd. Laparoscopic versus open inguinal hernia repair in pediatric patients: a systematic review. J Laparoendosc Adv Surg Tech A 2014;24:811-818.

8. Takahara H, Ishibashi $H$, Satoh $H$, et al. Laparoscopic surgery for inguinal lesions of pediatric patients. Paper presented at: Proceedings of 7th World Congress of Endoscopic Surgery; 2000 Jun 1-4; Singapore. p. 537-542.

9. Becmeur F, Philippe P, Lemandat-Schultz A, et al. A continuous series of 96 laparoscopic inguinal hernia repairs in children by a new technique. Surg Endosc 2004;18:1738-1741.

10. Rothenberg RE, Barnett T. Bilateral herniotomy in infants and children. Surgery 1955;37:947-950.

11. Öberg S, Andresen K, Rosenberg J. Etiology of inguinal hernias: a comprehensive review. Front Surg 2017;4:52.

12. Clarnette TD, Hutson JM. The genitofemoral nerve may link testicular inguinoscrotal descent with congenital inguinal hernia. Aust N Z J Surg 1996;66:612-617.

13. Anderson CC, Broadie TA, Mackey JE, Kopecky KK. Hydrocele of the canal of Nuck: ultrasound appearance. Am Surg 1995;61:959-961.

14. Zenitani M, Saka R, Sasaki T, et al. Safety and efficacy of laparoscopic percutaneous extraperitoneal closure for inguinal hernia in infants younger than 6 months: a comparison with conventional open repair. Asian J Endosc Surg 2019;12:439-445.

15. Levitt MA, Ferraraccio D, Arbesman MC, Brisseau GF, Caty MG, Glick PL. Variability of inguinal hernia surgical technique: a survey of North American pediatric surgeons. J Pediatr Surg 2002;37:745751.

16. Korkmaz M, Güvenç BH. Comparison of single-port percutaneous extraperitoneal repair and three-port mini-laparoscopic repair for pediatric inguinal hernia. J Laparoendosc Adv Surg Tech A 2018;28:337342.

17. Ho IG, Ihn K, Koo EJ, Chang EY, Oh JT. Laparoscopic repair of inguinal hernia in infants: comparison with open hernia repair. J Pediatr Surg 2018;53:2008-2012.

18. Liu J, Wu X, Xiu W, et al. A comparative study examining laparoscopic and open inguinal hernia repair in children: a retrospective study from a single center in China. BMC Surg 2020;20:244.

19. Dreuning K, Maat S, Twisk J, van Heurn E, Derikx J. Laparoscopic versus open pediatric inguinal hernia repair: state-of-the-art comparison and future perspectives from a meta-analysis. Surg Endosc 2019;33:3177-3191. 
20. Hoshino M, Sugito K, Kawashima H, et al. Prediction of contralateral inguinal hernias in children: a prospective study of 357 unilateral inguinal hernias. Hernia 2014;18:333-337.

21. Nataraja RM, Mahomed AA. Systematic review for paediatric metachronous contralateral inguinal hernia: a decreasing concern. Pediatr Surg Int 2011;27:953-961.

22. Miltenburg DM, Nuchtern JG, Jaksic T, Kozinetiz C, Brandt ML. Laparoscopic evaluation of the pediatric inguinal hernia: a metaanalysis. J Pediatr Surg 1998;33:874-879.

23. Ein SH, Njere I, Ein A. Six thousand three hundred sixty-one pediatric inguinal hernias: a 35-year review. J Pediatr Surg 2006;41:980-986.

24. Holcomb GW 3rd, Miller KA, Chaignaud BE, Shew SB, Ostlie DJ. The parental perspective regarding the contralateral inguinal region in a child with a known unilateral inguinal hernia. J Pediatr Surg 2004;39:480-482.

25. Antonoff MB, Kreykes NS, Saltzman DA, Acton RD. American
Academy of Pediatrics Section on Surgery hernia survey revisited. J Pediatr Surg 2005;40:1009-1014.

26. Brandt ML. Pediatric hernias. Surg Clin North Am 2008;88:27-43.

27. Moss RL, Hatch EI Jr. Inguinal hernia repair in early infancy. Am J Surg 1991;161:596-599.

28. Holcomb GW 3rd, Brock JW 3rd, Morgan WM 3rd. Laparoscopic evaluation for a contralateral patent processus vaginalis. J Pediatr Surg 1994;29:970-974.

29. Kang CH, Kim YJ, Kim KT. Initial experience with percutaneous internal ring suturing for indirect inguinal hernia in pediatric patients. J Minim Invasive Surg 2020;23:67-73.

30. Sumida W, Watanabe Y, Takasu H, Oshima K, Komatsuzaki N. Effects of insistent screening for contralateral patent processus vaginalis in laparoscopic percutaneous extraperitoneal closure to prevent metachronous contralateral onset of pediatric inguinal hernia. Surg Today 2016;46:569-574. 